\title{
Negative social events, stress, and health in Hong Kong
}

\author{
L P Shiu, W M Hui, S K Lam
}

\begin{abstract}
Study objective-To investigate the association, if any, between negative social events and physical illness.

Design-Comparison of major social events and indices of disease.

Setting-Hong Kong, 1962 to 1985.

Subjects-Patients treated in hospital for attempted suicide, coronary heart disease, and diabetes mellitus.

Measurements and main results-Major events were selected frrom the annual Hong Kong Year Book, and grouped in one of 18 categories by a panel of 10 assessors. Weights were assigned to each category according the likely stress produced. Individual events were then scored and multiplied by the category weights to produce an overall stress score from which a total stress score for each year was derived. Annual stress scores were then compared with hospital attendance rates for the three medical conditions.

Conclusions-The study has shown that: (1) the stress induced in the community by major negative social events in Hong Kong had been increasing; and (2) this stress is associated with attempted suicide but not with diabetes mellitus or coronary heart disease.
\end{abstract}

f Epidemiol Community Health 1993; 47: 181-185

The study of major life events and their relation with physical and psychological disorders has been popular since Holmes and Rahe proposed the social readjustment rating scale (SRRS). ${ }^{1}$ The basic assumption behind this type of research is that abrupt changes in life cause a high degree of stress that initiates readjustment or readaptation, which in turn makes a person more susceptible to diseases. This approach has shown that the major life events experienced by a person over a certain period (for example, six or 12 months) are related to the subsequent occurrence of a number of psychological and physical illnesses. ${ }^{2} 3$

Major negative social events may also be related to health from an epidemiological perspective. Major negative social events, such as an earthquake, usually affect a large number of people and cause them a considerable amount of stress. A relation with health might be seen in the spread of illnesses in the population that had been affected.

In fact, the relation between social events and personal life events is closer than it seems because a major social event can be viewed as a life event that is experienced by many people. Economic recession, for instance, is a major social event that drives many people out of their jobs: loss of employment is worth 47 life change units according to the SRRS!

Data have shown that the occurrence of some illnesses may increase and decrease relatively rapidly to an extent that cannot be explained by medical reasons. For example, Cassel ${ }^{4}$ noticed that tuberculosis was extremely common after the industrial revolution and began to decline after some 50 to 75 years. Between the two world wars, duodenal ulcer took the place of tuberculosis and became a widespread illness. Duodenal ulcer has been replaced in recent times by cancer, hypertension, and coronary heart diseases. Cassel suspected that changes in the social environment may explain these fluctuations. While tuberculosis may be more related to the socioeconomic situation, ${ }^{5}$ the pathogenesis of duodenal ulcer, hypertension, and coronary heart disease is believed to involve stress. $^{6-8}$ Negative social events that induce high levels of stress in a large number of people may have a role in the prevalence of these stress related illnesses.

It is evident that psychopathological disorders, such as attempted suicide or some other physical illnesses, do not follow a smooth trendconsiderable fluctuations occur from year to year. This is not easily explained by medical reasons such as improvements in technology and diagnosis, the development of preventive health care, or changes in smoking and dietary habits. Attempts have been made to relate these illness trends to environmental stressors such as crowding and noise levels. These endeavours have faced the same difficulty as before because the factors changed relatively steadily and the changes could not explain the abrupt fluctuations from year to year. The present study therefore looks to major negative social events that show fluctuation over the years for an explanation.

In fact, there are studies which have shown the effects of negative social events on health. For example, Baum et $a l^{9}$ investigated the effects of the Three Miles Island accident, while others have studied natural disasters and wars. The victims of these disasters suffered more illnesses than matched groups who had not had the same experiences. In addition, economic changes as well as unemployment have been throughly studied by Brenner et al, who showed significant correlations between unemployment and mortality, ${ }^{10}$ mental illnesses, ${ }^{11}$ and cardiovascular diseases. ${ }^{12} 13$ In contrast to these reports, the present study has included indiscriminately all major negative social events.

We have tried to quantify the amount of stress caused by the major negative social events that 
occurred in Hong Kong between 1962 and 1985. The occurrence of disorders that were both stress related (both physical and psychological) and unrelated during the same period were also collected. We intended to determine how far negative social events were related to the occurrence of stress related illnesses in Hong Kong.

Hong Kong is a city that has developed quickly over the past 100 years. Rapid modernisation and industrialisation have resulted in a highly stressful way of living. Between 1962 and 1985 Hong Kong has had to face major crises and negative social events. For example, in 1963-4, there was water rationing which limited the water supply to four hours every four days. In 1967, there were large scale riots in various parts of Hong Kong. At that time, many people fled to other countries and the stock and property markets slumped. More recently, massive influxes of immigrants from China (both legal and illegal) and boat refugees from Vietnam have placed increased social pressures on the population. As a commercial centre, Hong Kong has continually been confronted with wide fluctuations in the international monetary markets and trade restrictions. Since the early 1980s almost everyone living in Hong Kong has been troubled by what will happen in 1997 . Hong Kong has been under British rule since 1842 , but according to the 'Joint Declaration' made by the Peoples' Republic of China and the British government, it will become part of China after 1 July 1997. Many Hong Kong people are worried about the stability and prosperity of their society and this has resulted in large scale migration to other countries. Hong Kong therefore constitutes a unique environment for the study of these negative social events.

Negative social events have certainly brought a considerable amount of stress to many people living in Hong Kong. Fortunately, they did not occur every year, but many have been sudden, unique, and powerful events that have affected large numbers of people. Lazarus and Cohen ${ }^{14}$ have labelled these sorts of events 'cataclysmic phenomena' but their implications for health have not been studied systematically. We look at the relationship between 'cataclysmic phenomena' and the prevalence of attempted suicide, coronary heart disease and diabetes mellitus in the population.

Because attempted suicide is often found to be preceded by major life events, ${ }^{15}$ the occurrence figures for Hong Kong are expected to correlate

The 18 categories of events and their respective weights highly with negative social events. Stress has also been implicated in the pathogenesis of coronary heart disease. The degree of association, however, is expected to be less than that for suicide, as other factors such as smoking, raised serum cholesterol and lipid concentrations and hypertension ${ }^{16-18}$ have been identified as major risk factors for coronary heart disease. With regard to diabetes mellitus, though there have been reports relating this disorder to stress, it is generally considered to play only a minor role, ${ }^{19}$ and its prevalence is not expected to correlate significantly with the negative social events.

\section{Methods}

PRESS CUTTINGS

Important negative events that occurred in Hong Kong between 1962 and 1985 were selected from the Hong Kong Year Book, ${ }^{20}$ which is published annually by Wah Kiu Yat Po, one of the biggest Chinese newspaper agencies in Hong Kong. In the early 1960s, the events were divided into categories (for example, political, economic, etc) and were listed in chronological order. An event was named, described in a short sentence and the date of occurrence was provided. After the 1970 s, however, events were no longer arranged chronologically but formed into clusters with a brief description of each cluster of events. Perhaps this reflects the fact that life has become more complicated in Hong Kong and a listing of all events for every single day of the year would be unmanageable.

The authors were astonished that fewer events were listed for the early 1960 s than the 1980 s. As a control study, the 1965 and 1966 editions of the Sunday South China Morning Post, a major local English newspaper which provided a weekly summary of important events, were reviewed. It was found that the Hong Kong Year Book adequately included all the major events of those two years.

The first author selected events according to the criterion that they must either bring about major changes in society or affect the wellbeing of a substantial portion of the population. In addition, eight independent judges (two men, aged 20-26 years), blind to the purpose of the study, were paid US $\$ 10$ (approximately) to read through all the material to check if any events that satisfied the above criteria had been omitted. A summary of major news was then compiled.

\section{RATING}

Ten judges (two men, aged 19-36 years) scored the events. They were paid US\$10 (approximately) for their participation. Events were firstly divided into 18 categories and then arranged in yearly order (table). The judges were asked to weigh the relative importance of each category in the total amount of stress suffered by the people of Hong Kong. They were asked to distribute 100 points over the 18 categories to indicate the importance of each, and these were the weight scores. Secondly, the judges were asked to rate the severity of the individual events under each catagory over a period of 24 years. The overall scoring of all events under each category was then rated against a five point scale and was defined as 
the severity score. The level of stress brought about by each category of events was taken as the weight score $x$ the severity score. Finally, a total stress score for each year was derived by summing the 18 sub scores. It was recognised that judges might give a higher severity rating to those events they had experienced directly and that this would be particularly serious in cases where the events affected only part of the population. To compensate for the influence of personal experiences, the judges were given a brief description of the events wherever possible and were told to base their judgements on the descriptions and to avoid thinking about their own experiences.

\section{VALIDATION FROM ANOTHER INDEX}

As a validation of the stress scores, the crime rates for the 24 years were also collected. The crime rate was the number of crimes reported to the police department $\mathrm{p} / 100000$ population per year. Attempts had been made to collect unem-
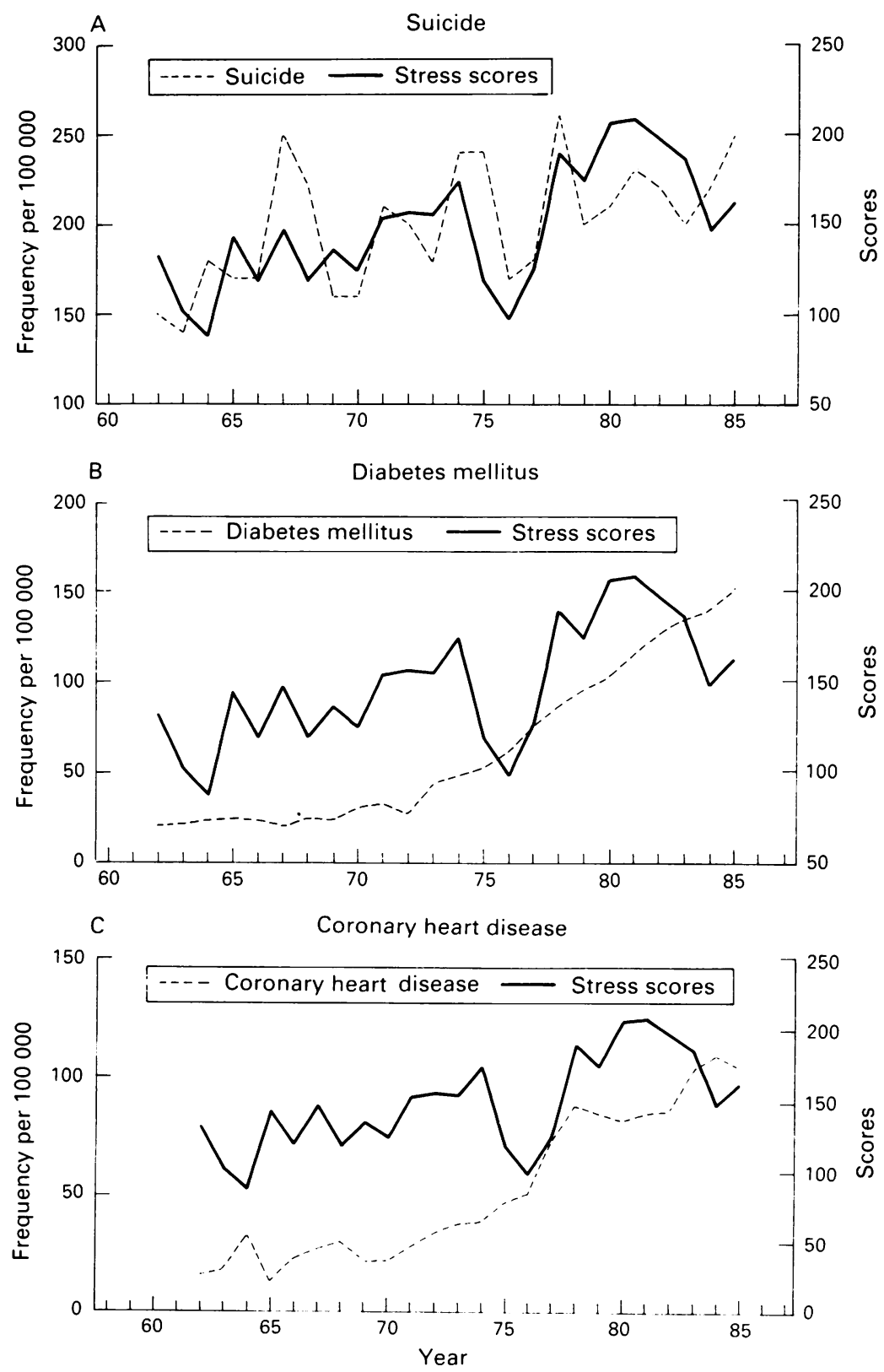

Inter-relationship between the stress scores and the incidence of $(A)$ attempted suicide, $(B)$ diabetes mellitus and, (C) coronary heart disease ployment and divorce figures but the Hong Kong government did not keep accurate records until 1976 and 1972 respectively so these figures were not used for validation.

\section{COLLECTION OF HEALTH INDICES}

Figures were obtained from the annual report published by the Medical and Health Department of Hong Kong. ${ }^{21}$ The number of patients treated in all government and government assisted hospitals (which served over $85 \%$ of the population of Hong Kong) with a diagnosis of attempted suicide, coronary heart disease, or diabetes mellitus were determined. The diagnosis of coronary heart disease was based on clinical findings, electrocardiogram, cardiac enzymes with or without exercise test, and coronary angiograms. Diabetes mellitus was diagnosed if the fasting venous blood sugar concentration was $\geqslant 8 \mathrm{mmol} / 1$ or if after glucose challenge $(75 \mathrm{~g})$ the glucose concentration at two hours was $\geqslant 11.0 \mathrm{mmol} / 1$. The respective relation between these diseases and the stress scores for each year was the point of interest.

\section{Results}

STRESS SCORE

The ratings given to the 18 categories of events listed in the table by the 10 judges were averaged. The agreement among judges was fairly strong. The inter-rater correlation coefficients (Spearman's $r$ ) ranged from 0.37 to 0.94 with a mean of 0.69 for the weight rating, and ranged from 0.53 to 0.95 with a mean of 0.65 for the severity rating. All except 2 ( $p<0.07$ for both) was significant at the 0.05 level. The Spearman's $r$ for the stress scores and crime rates was $0.69(\mathrm{p}<0.001)$. Kappa statistics $^{22}$ were also used to assess the agreement between judges in the overall classification of events. The mean Kappa was 0.27 (range 0.06$0 \cdot 72$ ). When tranformed to a $z$-score, $z=4 \cdot 78$, and this is significant at a p value of 0.05 , indicating that there was significant agreement among the judges.

\section{STRESS AND DISEASES}

The stress scores and the number of patients with the various diseases are plotted in the figure. There is considerable correlation between stress scores and attempted suicide, and stress scores and coronary heart disease. The curve representing diabetes mellitus rises smoothly over the years showing no remarkable peaks and troughs. The stress level curve, however, fluctuates greatly over the years. To show that the occurrence frequencies of the illnesses were sensitive to the level of stress, the linear trends found in these curves should be partialled out in the analysis. The linear trend probably indicates that as the development of the society progressed, the amount of stress as well as occurrence of illnesses increased. It might mask the real relation between the level of stress and the occurrence of illnesses. Therefore, an analysis using the following model was carried out:

\section{$\mathbf{Y t}=\mathrm{X} 1 \mathbf{Y} \mathrm{t}-1+\mathrm{B} 1 \mathbf{X} \mathrm{t}-1+\mathrm{BoX} \mathrm{t}+\mathbf{A t}$}

Yt was defined as the dependent variable at time $t$, while YT-1 was the value of $\mathbf{Y}$ at time $t-1$, which 
was here defined as $\mathbf{Y}$ in the previous year. $\mathbf{X}$ was the independent variable, which was the stress score in our study. X1, B1, and Bo were the weights given to the variables. At was the constant. This analysis regressed the value of $\mathbf{Y}$ at time $t$ in terms of $\mathbf{Y}$ in the previous year and $\mathbf{X}$ at time $t$ and $\mathbf{X}$ in the previous year. Since the values of both $\mathbf{X}$ and $\mathbf{Y}$ in the previous year were taken into consideration, the linear trends found in both $\mathbf{X}$ and $\mathbf{Y}$ would be taken care of. The coefficient Bo would indicate the relationship between $\mathbf{X}$ and $\mathbf{Y}$ at time $t$, given the linear trends partialled out. Thus the direction of Bo (that is, whether positive or negative) indicates the direction of the association, while its value indicates the strength of the association.

The Bo values for stress in relation to attempted suicide, coronary heart disease, and diabetes mellitus were $0.54(p<0.05),-0.08(p>0.05)$, and $0.01(p>0.05)$ respectively, and the Bo for the crime rate is $0.60(p<0.01)$. Bo is significant at 0.05 level if $\mathrm{Bo}-2$ standard errors of $\mathrm{Bo}>0$ (for $\mathrm{Bo}>0$ ); or if $\mathrm{Bo}+2$ standard errors of $\mathrm{Bo}<0$ (for $\mathrm{Bo}<0$ ). Because these coefficients were obtained with the scores standardised before entering into the equation, it is iegitimate to compare them. It is clear that the relationship between stress and attempted suicide was the strongest. The correlation between stress and diabetes mellitus, which yielded a coefficient of 0.60 in the Spearman test of correlation, diminished when the linear trend was partialled out. The relation between stress and coronary heart disease was weak according to this model; although it was stronger than that between stress and diabetes mellitus, the direction was negative.

\section{Discussion}

This study showed that the stress induced in society by the major negative social events that have occurred in Hong Kong has been increasing. The validity of the stress scores was verified by the significant correlation with the crime rate, which is an objective quantitative social index. The study also showed that attempted suicide increased when there was a parallel increase in the amount of stress in the community. This was expected because suicide is often regarded as a psychopathology that is closely related to stress. A number of studies have shown that suicides were preceded by one or more major negative personal life events, ${ }^{23}$ especially among depressed patients. ${ }^{24}$ The present study found that this relation also existed in the community.

The relation between negative social events and physical health, however, was not as strong. As predicted, there was no observable relation between major negative social events and diabetes mellitus once the linear trends were partialled out. Although there was a significant correlation between coronary heart disease and stress using the Spearman rank correlation, the association diminished when the linear trends in both variables were partialled out. This contrasts with some studies which reported a positive relation on an individual basis. ${ }^{25}$ The relation between stress and physical illnesses may depend on other variables, so that it fails to reach significance at the epidemiological level. This is consistent with the observation that not every individual felt sick when confronted with the same negative event. Nevertheless, it should be noted that the present finding neither supports nor refutes the relation between stress and coronary heart disease found at an individual level. It simply shows that the negative social events and numbers of attempted suicides in the same community were related, while no such relation was found between social events and coronary heart disease.

A major difficulty in this kind of research is how to quantify the amount of stress caused by the major negative social events. We tried to tackle this problem by following the pioneering work of Holmes and Rahe. ${ }^{1}$ Given the resemblance between personal life events and social events, research in this direction may shed new light on epidemiological studies. Major social events differ from ordinary indices of community stress such as crime, alcoholism, divorce, and juvenile delinquency in their sensitivity to time change. While major social events usually happen suddenly the indices for other community stresses generally follow a smooth trend. It is unlikely therefore that ordinary community stresses account for the sudden fluctuations in the occurrence of health problems.

Moreover, even if there is a causal relation between stress and illnesses, these ordinary indices of community stress cannot be considered as 'causes' because they themselves are probably the consequences of stress. Major negative social events, however, are far less likely to be the consequences of stress and could assume a causal role if there were indeed a relation.

Before useful conclusions can be drawn, however, the time lag between the occurrence of an event and its expected consequence on health should be well understood. Previous research has tried to fit the two variables using different time lags, and decided on the one which gave the best fit. Although this seems to be the only method presently available, it did not give a definite time lag that applied to different studies. ${ }^{26}$ Brenner and Mooney, ${ }^{27}$ for example, used a time lag of $0-5$ years in studying unemployment and cardiovascular disease mortality in Britain between 1955 and 1976 , while Antoni ${ }^{28}$ reported a six month lag for life event measurements and illnesses recorded in medical files. The present study made no adjustment in time lag when matching the variables. But it seems that the relation between social events and attempted suicide still holds at no time lag (in terms of years). This problem is not limited to the study of major social events but should also be a concern for personal life events research. Further investigation of time lag is relation to major events and their consequences is needed.

L Shiu is now at the University of California, San Diego. the study was supported by the Peptic Ulcer Research Fund (311/041/0372), University of Hong Kong. The authors are grateful to Dr D Y F Ho, Dr H C C Hui, and Dr D Lam for their advice and help concerning the derivation of the stress scores. Thanks also to $\mathrm{Dr} W \mathrm{~K} \mathrm{Li}$ for his expert advice on statistical analysis, and Karen Chan and April Wong for secretarial help. 
1 Holmes TH, Rahe $\mathrm{RH}$. The social readjustment rating scale. f Psychosom Res 1967; 11: 213-8.

2 Krantz DZ, Grunberg NE, Baum A. Health psychology. Ann Rev Psychol 1985; 36: 349-83.

3 Rabkin JG, Streuning EL. Life events, stress, and illness. Science 1976; 194: 1013-20.

4 Cassel J. Physical illness in response to stress. In: Levine S, Scotch NA, eds. Social Stress. Chicago: Aldine, 1970.

5 Leff A, Lester TW, Addington WW. Tuberculosis: a chemotherapeutic triumph but a persistent socio-economic problem. Arch Int Med 1979; 130: 1375-7.

6 Feldman M, Walker P, Green JL, Weingarden K. Life events stress and psychological factors in men with Peptic ulcer stress and psychological factors in men with
disease. Gastroenterology 1986; 91: 1370-8.

7 disease. Gastroenterology 1986; 91: 1370-8.
7 Sever PS, Poulter NR. A hypothesis for the pathogenesis of essential hypertension: the initiating factors. $\mathcal{f}$ Hypertens 1989; 7 (suppl): 9-12.

1989; 7 (suppl): $9-12$.
8 Jenkins CD. Recent evidence supporting psycholigic and social risk factors for coronary disease. N Engl F Med 1976; 294: 987-1033-8.

9 Baum A, Gatchel R, Shaeffer M. Emotional behavioural, and physiological effects of chronic stress at Three Miles Island. f Consult Clin Psychol 1983; 51: 565-72.

10 Brenner MH, Mortality and the national economy: a review and the experience of England and Wales, 1936-1976. Lancet 1979; 15: 568-73.

11 Brenner MH. Mental illness and the economy. Cambridge MA: Harvard University Press, 1973.

12 Brenner MH. Economic changes and heart disease mortality. Am f Public Health 1971; 61: 606-11.

13 Brenner MH, Mooney A. Economic change and sex-specific cardiovascular mortality in Britain 1955-1976. Soc Sci Med 1982; 16: 431-42.

14 Lazarus RS, Cohen JB. Environmental stress. In: Altman I, Wohlwill JF eds. Human behaviour and the environment: current theory and research. New York: Plenum, 1977

15 Lo WH, Leung TM. Suicide in Hong Kong. Aust $N Z \mathcal{J}$ Psychiatry 1985; 19: 287-92.
16 Carlson LA, Bottiger LE, Anfeldt PE. Risk factors for myocardial infarction in the Stockholm Prospective Study. Acta Medica Scandinavia 1979: 206; 351-60.

17 Dawber TD. The Framingham Study. Cambridge MA: Harvard University Press, 1980.

18 Dwyer J, Hetzel BS. A comparison of trends of coronary heart disease mortality in Australia, USA and England and Wales with reference to three major risk factors hypertension, smoking and diet. Int $\mathcal{F}$ Epidemiol 1980; 9 . $65-71$.

19 Roger RV, Foster DW. Diabetes mellitus. In: Wilson JD, Foster DW eds. Williams' Textbook of endocrinology. New York: W B Saunders, 1985: 1018-1107.

20 Hong Kong Year Book. Volumes for 1962-1985. Hong Kong: Wah Kiu Yat Po: 1962-1985.

21 Annual Report of the Medical and Health Department of Hong Kong, Volumes for 1962-1985. Hong Kong: The Medical and Health Department of Hong Kong: 1962-1985.

22 Wickensam TD. Multiway contingency tables analysis for the social sciences. Hillsdale NJ, Erlbaum: 1989.

23 Paykel ES, Prusoff BA, Myers JK. Suicide attempts and recent life events. Arch Gen Psychiatry 1975; 32: 327-33.

24 Slater J, Depue RA. The contribution of environmental events and social support to serious suicide attempts in primary disorders. 7 Abnorm Psychol 1981; 90: 275-85.

25 Roseman RH, Brand RJ, Jenkins CD, Friedman M, Straus $\mathrm{R}$. Coronary heart disease in the western collaborative group study: final follow-up experience of $8 \%$ years. $7 A M A 1975$ 223: 872-7.

$26 \mathrm{Kasl}$ SV. Mortality and the business cycle: some questions about research strategies when utilizing macro-social and ecological data. Am f Public Health 1979; 69: 784-8.

27 Brenner MH, Mooney A. Unemployment and health in the context of economic change. Soc Sci Med 1983; 17: 112538.

28 Antoni MH. Temporal relationship between life events and two illness measures: a cross-lagged panel analysis. fournal of Human Stress 1985; 11: 21-6. 